\title{
Dynamics of Antibodies to Various Antigens of the SARS-CoV-2 Coronavirus in Patients with Confirmed COVID-19 Infection
}

\author{
A. V. Aleshkin ${ }^{1}$, L. I. Novikova ${ }^{1}$, S. S. Bochkareva ${ }^{1}$, S. Yu. Kombarova ${ }^{1}$, \\ Yu. S. Lebedin ${ }^{3}$, A. M. Vorob'ev', E. R. Mekhtiev', E. R. Zul'karneev', \\ A. I. Laishevtsev' ${ }^{2}$ and A. V. Karaulov ${ }^{1}$
}

Translated from Byulleten'Eksperimental'noi Biologii i Meditsiny, Vol. 171, No. 2, pp. 196-199, February, 2021 Original article submitted October 19, 2020

\begin{abstract}
The presence of IgG and IgM antibodies in the venous blood of 76 patients with confirmed COVID-19 infection was determined by ELISA using Russian test systems. Different levels of IgM antibodies to N-protein and receptor binding domain of the Spike protein (RBD) were revealed. The dynamics of IgG antibodies to the whole virion antigen and recombinant antigens showed high values on weeks $4-5$ of the disease. The level of IgG antibodies to Nprotein remained low throughout the observation period. The characteristic dynamics of IgG measured using test systems with sorbed whole virion or recombinant spike proteins reflects the duration of the disease.
\end{abstract}

Key Words: antibodies; dynamics; humoral immunity; SARS-CoV-2; COVID-19

The infection caused by SARS-CoV-2 coronavirus (COVID-19), which began with an outbreak in China at the end of 2019, has spread throughout the world in a few months, becoming a pandemic. Detection of viral RNA in biological material (real-time RT-PCR) is a standard laboratory diagnostic method for COVID-19. At the same time, the study of humoral immunity to SARS-CoV-2 by various immunochemical methods also has undoubted diagnostic value when examining COVID-19 patients or persons suspected of being infected with this coronavirus.

The aim of this study was to detect antibodies to different antigens of the SARS-CoV-2 virus and to determine the time of appearance and duration of persistence of different antibodies in patients with confirmed COVID-19 infection.

${ }^{1}$ G. N. Gabrichevsky Moscow Research Institute for Epidemiology and Microbiology, Federal Service for Surveillance on Consumer Rights Protection and Human Wellbeing, Moscow, Russia; ${ }^{2}$ Center of Food Biotechnological Processing, Institute of Applied Microecology LLC, Moscow, Russia; ${ }^{3}$ XEMA Co., Ltd., Moscow, Russia. Address for correspondence: vorobjew.alex2010@yandex.ru. A. M. Vorob'ev

\section{MATERIALS AND METHODS}

IgG and $\operatorname{IgM}$ antibodies were tested in the venous blood of 76 patients with the presence of the SARS$\mathrm{CoV}-2$ virus confirmed by PCR and/or CT (a total of 166 samples). Of 76 patients, 61 were examined in dynamics (from 38 patients, the samples were taken twice at different terms of the disease, 3 times from 18 patients, 4 times from 4 patients, and 5 times from 1 patient, i.e. from 2 to 5 survey points were analyzed). Blood serum samples were kindly provided by the N. I. Pirogov National Medical and Surgical Center, Ministry of Health of the Russian Federation, where patients were treated for Covid-19 infection in May 2020. The data on the duration of the disease were taken from patient's medical records.

IgG antibodies to SARS-CoV-2 were detected by ELISA using Russian diagnostic test systems with whole-virion SARS-CoV-2 particles (SARS-CoV-2ELISA-Vector, State Research Center of Virology and Biotechnology Vector), with recombinant nucleocapsid protein (N-protein) (ELISA anti-SARS-CoV-2 
IgG, State Research Center for Applied Microbiology and Biotechnology), with recombinant full-length trimerized glycoprotein (Spike-protein) (SARS-CoV-2IgG-ELISA-BEST, Vector-Best), and with recombinant RBD protein (receptor binding domain of the Spike protein) (SARS-CoV-2-IgG-ELISA, National Medical Research Center of Hematology, Ministry of Health of the Russian Federation) adsorbed in the plate wells. For detection of IgM antibodies to SARS-CoV-2, we used Russian test systems based on the indirect version of ELISA with antibodies to human IgM immobilized in the wells and detection of specific antibodies using conjugates containing virus antigens: N-nucleocapsid protein (SARS-CoV-2-IgMELISA-BEST, Vector-Best) or RBD (receptor binding domain of the Spike protein) (SARS-CoV-2-IgM-ELISA, XEMA; a special conjugate containing RBD was kindly provided by the manufacturer).

The results were expressed as a positivity coefficient (PC), i.e. the ratio of the optical density of the sample to the critical optical density calculated in each analysis. Depending on the test system used, samples were considered positive at $\mathrm{PC}>1.1-1.2$ and negative at $\mathrm{PC}<0.8-0.9$. Intermediate values indicated a dubious or undefined result (gray zone).

\section{RESULTS}

In the early follow-up period (up to day 4), IgM and IgG antibodies were not detected, with the exception of antibodies of both classes to N-protein and IgG antibodies to Spike protein trimer (Table 1). However, it should be noted that the level of IgM antibodies was recorded in the "gray zone" (1.0-1.1), and detection of IgG antibodies to trimerized glycoprotein in one patient (PC 1.7) most likely indicates an earlier infection.
On days 5-9 (on average, the first week), antibodies of both IgM and IgG classes were detected in about half of the patients by all test systems used, with the exception of IgM antibodies to RBD that were detected in a $1 / 3$ of the patients. The mean PC of IgM antibodies to N-protein was 3.8; IgM to RBD just appeared (mean PC was slightly surpassed 1.2). The highest level of IgG antibodies was recorded to the trimerized glycoprotein (PC 5.1) and the lowest to the N-protein (PC 1.4).

Starting from days 10-19 (on average, the second week) up to days 30-39 (on the average, the fifth week), IgM antibodies to N-protein were recorded in almost all patients (PC 8.0). At the same time, IgM antibodies to RBD were detected not in all patients (in $73.2 \%$ cases on days $10-19$, in $79.5 \%$ on days 20-29, and in 55.6\% on days 30-39), and PC did not exceed 2.4. All studied IgG antibodies were detected in $92.3-100 \%$ cases during the same observation periods; PC of antibodies to the N-protein remained at the level of 3.6-3.9, while antibodies to the whole virion and spike proteins reached much higher values (PC 9.6-14.8).

At later terms (days 40-49 and 50-53, the 7th-8th weeks), IgG antibodies to all studied antigens were still detected. At the same time, the level of specific IgG to $\mathrm{N}$-protein remained within the same values (PC 3.1-3.4), to the whole virion antigen and RBD decreased to 6.6-6.1 and 7.6-6.9, respectively, and to the trimerized glycoprotein remained high (PC 9.611.1). By the 7th-8th weeks of observation, IgM antibodies began to disappear from the bloodstream. Thus, IgM antibodies to RBD and N-protein were detected only in 25 and $50 \%$ patients, respectively. It should be noted that in 2 of 76 patients examined, antibodies did not develop at all or were produced weakly. In one patient of these (blood serum samples were taken

TABLE 1. The Frequency of Detection of IgG and IgM Antibodies in Patients at Different Observation Periods

\begin{tabular}{|c|c|c|c|c|c|c|c|c|c|c|c|c|}
\hline \multirow{3}{*}{$\begin{array}{l}\text { Observation } \\
\text { period }\end{array}$} & \multicolumn{8}{|c|}{ IgG antibodies to various SARS-CoV-2 antigens } & \multicolumn{4}{|c|}{$\begin{array}{l}\text { IgM antibodies to various } \\
\text { SARS-CoV- } 2 \text { antigens }\end{array}$} \\
\hline & \multicolumn{2}{|c|}{$\mathrm{N}$-protein } & \multicolumn{2}{|c|}{$\begin{array}{l}\text { inactivated } \\
\text { whole virion }\end{array}$} & \multicolumn{2}{|c|}{$\begin{array}{l}\text { trimerized glyco- } \\
\text { protein (Spike) }\end{array}$} & \multicolumn{2}{|c|}{ RBD } & \multicolumn{2}{|c|}{$\mathrm{N}$-protein } & \multicolumn{2}{|c|}{ RBD } \\
\hline & abs. & $\%$ & abs. & $\%$ & abs. & $\%$ & abs. & $\%$ & abs. & $\%$ & abs. & $\%$ \\
\hline $3-4$ days $(n=6)$ & 1 & 16.7 & 0 & 0 & 1 & 16.7 & 0 & 0 & 1 & 16.7 & 0 & 0 \\
\hline $5-9$ days $(n=29)$ & 15 & 51.8 & 14 & 48.3 & 16 & 55.2 & 15 & 51.8 & 14 & 48.3 & 10 & 34.5 \\
\hline $10-19$ days $(n=56)$ & 56 & 100.0 & 55 & 98.2 & 56 & 100.0 & 52 & 92.9 & 56 & 100.0 & 41 & 73.2 \\
\hline $20-29$ days $(n=39)$ & 38 & 97.4 & 36 & 92.3 & 38 & 97.4 & 37 & 94.9 & 38 & 97.4 & 31 & 79.5 \\
\hline 30-39 days $(n=9)$ & 9 & 100.0 & 9 & 100.0 & 9 & 100.0 & 9 & 100.0 & 9 & 100.0 & 5 & 55.6 \\
\hline 40-49 days $(n=5)$ & 4 & 80.0 & 4 & 80.0 & 4 & 80.0 & 4 & 80.0 & 3 & 60.0 & 2 & 40.0 \\
\hline $50-53$ days $(n=4)$ & 3 & 75.0 & 4 & 100.0 & 4 & 100.0 & 4 & 100.0 & 2 & 50.0 & 1 & 25.0 \\
\hline
\end{tabular}


on days 15 and 22 of the disease), only specific IgM to $\mathrm{N}$-protein were detected, while IgG antibodies to neither N-protein, nor spike proteins, nor whole virion were detected in these terms; in another patient (serum samples were taken only on days 43 and 53 of the disease), no IgM antibodies were detected (we do not known whether they were at an earlier stage of the disease), while IgG antibodies were detected only on day 53 to the whole virion antigen (PC 2.9), to the trimerized spike glycoprotein (PC 5.5), and RBD (PC 1.1), but not to N-protein.

Different dynamics of the production of $\mathrm{IgG}$ and IgM antibodies to $\mathrm{N}$-protein of the nucleocapsid and to $\mathrm{RBD}$, the main domain of the glycoprotein of the spike of the virus, was observed (Fig. 1). Both IgM antibodies were detected starting from the first week of the disease; then, the level of IgM antibodies to RBD did not reach high values (PC remained at the level of 1.2 to 3.2 and decreased to 1.6 by the 8 th week), while the level of IgM antibodies to N-protein sharply increased from zero values to 8.0 (the 3rd week of the disease). Over the entire observation period, the concentration of these specific IgM remained high and decreased only by the 8th week to PC 3.3. The dynamics of specific IgG production was opposite. The concentration of IgG antibodies to RBD increased sharply starting from the first week of the disease from PC 2.4 to PC 11.1 by the 5 th week, while the level of $\mathrm{IgG}$ antibodies to N-protein increased smoothly and PC did not exceed 4.0. The dynamics of the production of $\operatorname{IgM}$ and IgG antibodies to the $\mathrm{N}$-protein of the nucleocapsid was consistent published data $[2,6,7]$.

The degree of elevation of concentration of IgG antibodies measured by the test systems with sorbed whole virion or recombinant spike proteins allows us to determine the duration of the disease (i.e., the period from the moment of infection), which confirms the need for diagnostic serological testing of patients with COVID-19 [6], while the level of IgG antibodies to the $\mathrm{N}$-protein remains at the same low level throughout the disease.

We also revealed a correlation between the levels of IgG antibodies to the whole virion antigen and recombinant spike antigens (trimerized glycoprotein and $\mathrm{RBD}$ ); under conditions of pronounced response to the virus, all three variants of diagnostic test systems can be used to assess the antiviral humoral immunity. However, according to test manufacturers, the test system based on the whole virion antigen can also detect antibodies to seasonal coronaviruses, which can lead to false positive results when analyzing samples for antibodies to SARS-CoV-2. In this regard, we consider that at low PC values recorded using the whole virion system, it is necessary to analyze samples in systems with sorbed recombinant antigens.

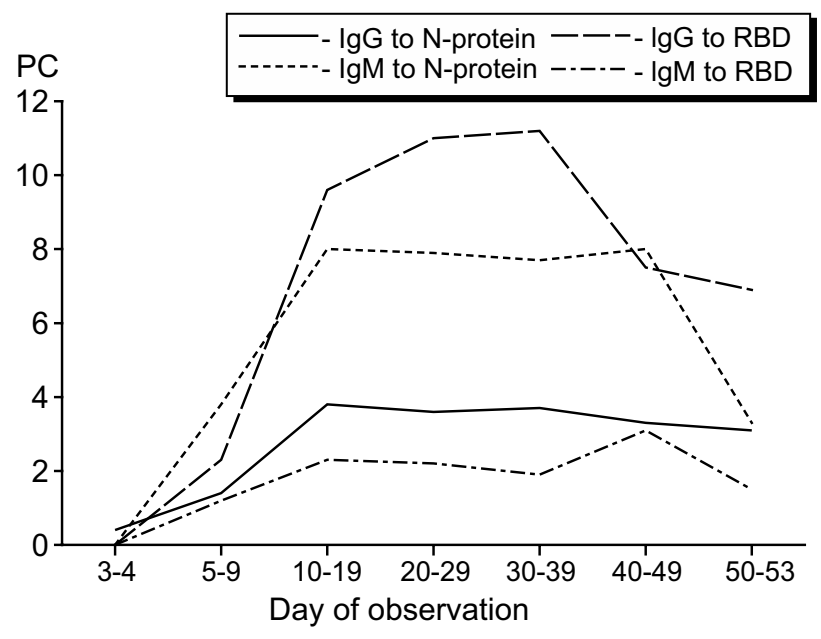

Fig. 1. Mean levels of $\lg M$ and $\lg G$ antibodies to $\mathrm{N}$-protein and RBD of SARS-CoV-2 at different terms of observation.

The detection of IgG antibodies to spike protein is of great importance in light of published data that these antibodies have a protective function during infection with SARS-CoV-2 and neutralize the effect of the virus [5], while spike protein is considered as the candidate for the design of appropriate vaccines [1,3-5].

During the first 5 days after infection, no IgM and IgG antibodies to all studied antigens of the SARS$\mathrm{CoV}-2$ virus were detected in the blood serum of patients with confirmed COVID-19 infection. Seroconversion of both $\operatorname{IgM}$ and $\mathrm{IgG}$ was observed during the first week of the disease, which was demonstrated by all 6 ELISA test system.

The dynamics of IgM antibodies to N-protein and RBD was similar, but the level of antibodies was different. IgM antibodies to RBD appeared during the first week of the disease, but did not reach high values, whereas the level of IgM antibodies to the N-protein sharply increased from zero to high PC values over a short period (by the 3 rd week of the disease). The concentration of these specific IgM remained high throughout the observation period and decreased only by the 8 th week.

The dynamics of IgG antibodies to the whole virion antigen and to the recombinant antigens of the spike was similar, with the maximum values of indicators revealed by $4-5$ weeks of observation. The dynamics of IgG antibodies to the N-protein differed from the dynamics of antibodies to the whole virion antigen and recombinant spike antigens: a slight increase in the concentration by the 1 st week and a low level throughout the observation period (8 weeks).

Different dynamics of the production of IgG and IgM antibodies to nucleocapsid N-protein and to RBD, the main domain of the virus spike-protein, was noted. 
The dynamics of antibodies to N-protein was characterized by a sharp rise and high level of IgM antibodies, while the level of IgG antibodies grew more smoothly and did not reach high values. For antibodies to RBD, the opposite pattern was observed.

The characteristic dynamics of the level of $\operatorname{IgG}$ antibodies measured using test systems with adsorbed whole virion or recombinant spike proteins (but not with $\mathrm{N}$-protein) reflects the duration of the disease (i.e., the period from infection), which has diagnostic value.

The authors are grateful to O. A Karpov, A. A. Pulin, and O. A. Orlova (N. I. Pirogov National Medical and Surgical Center, Ministry of Health of the Russian Federation) for kindly presented clinical material.

\section{REFERENCES}

1. Amanat F, Krammer F. SARS-CoV-2 vaccines: status report. Immunity. 2020;52(4):583-589. doi: 10.1016/j.immuni.2020.03.007

2. Bundschuh C, Egger M, Wiesinger K, Gabriel C, Clodi M, Mueller T, Dieplinger B. Evaluation of the EDI enzyme linked immunosorbent assays for the detection of SARS-CoV-2
IgM and IgG antibodies in human plasma. Clin. Chim. Acta. 2020;509:79-82. doi: 10.1016/j.cca.2020.05.047

3. Salvatori G, Luberto L, Maffei M, Aurisicchio L, Roscilli G, Palombo F, Marra E. SARS-CoV-2 Spike protein: an optimal immunological target for vaccines. J. Transl. Med. 2020;18(1):222. doi: 10.1186/s12967-020-02392-y

4. Tai W, He L, Zhang X, Pu J, Voronin D, Jiang S, Zhou Y, $\mathrm{Du}$ L. Characterization of the receptor-binding domain (RBD) of 2019 novel coronavirus: implication for development of RBD protein as a viral attachment inhibitor and vaccine. Cell. Mol. Immunol. 2020;17(6):613-620. doi: 10.1038/s41423-0200400-4

5. Walls AC, Park YJ, Tortorici MA, Wall A, McGuire AT, Veesler D. Structure, function, and antigenicity of the SARSCoV-2 Spike glycoprotein. Cell. 2020;181(2):281-292.e6. doi: 10.1016/j.cell.2020.02.058

6. Xiang F, Wang X, He X, Peng Z, Yang B, Zhang J, Zhou Q, Ye H, Ma Y, Li H, Wei X, Cai P, Ma WL. Antibody detection and dynamic characteristics in Patients with COVID-19. Clin. Infect. Dis. 2020;71(8):1930-1934. doi: 10.1093/cid/ciaa461

7. Zhao J, Yuan Q, Wang H, Liu W, Liao X, Su Y, Wang X, Yuan J, Li T, Li J, Qian S, Hong C, Wang F, Liu Y, Wang Z, He Q, Li Z, He B, Zhang T, Fu Y, Ge S, Liu L, Zhang J, Xia N, Zhang Z. Antibody response to SARS-CoV-2 in patients of novel coronavirus disease 2019. Clin. Infect. Dis. 2020;71(16):2027-2034. doi: 10.1093/cid/ciaa344 